\title{
Coronal heating and flaring in QSLs
}

\author{
Guillaume Aulanier \\ Observatoire de Paris, LESIA, CNRS, UPMC, Universit Paris Diderot, \\ 5 place Jules Janssen, 92190 Meudon, France \\ email: guillaume.aulanier@obspm.fr
}

\begin{abstract}
Quasi-Separatrix Layers (QSLs) are 3D geometrical objects that define narrow volumes across which magnetic field lines have strong, but finite, gradients of connectivity from one footpoint to another. QSLs extend the concept of separatrices, that are topological objects across which the connectivity is discontinuous. Based on analytical arguments, and on magnetic field extrapolations of the Sun's coronal force-free field above observed active regions, it has long since been conjectured that QSLs are favorable locations for current sheet (CS) formation, as well as for magnetic reconnection, and therefore are good predictors for the locations of magnetic energy release in flares and coronal heating. It is only up to recently that numerical MHD simulations and solar observations, as well as a laboratory experiment, have started to address the validity of these conjectures. When put all together, they suggest that QSL reconnection is involved in the displacement of EUV and SXR brightenings along chromospheric flare ribbons, that it is related with the heating of EUV coronal loops, and that the dissipation of QSL related CS may be the cause of coronal heating in initially homogeneous, braided and turbulent flux tubes, as well as in coronal arcades rooted in the slowly moving and numerous small-scale photospheric flux concentrations, both in active region faculae and in the quiet Sun. The apparent ubiquity of QSL-related CS in the Sun's corona, which will need to be quantified with new generation solar instruments, also suggests that QSLs play an important role in stellar's atmospheres, when their surface radial magnetic fields display complex patterns.
\end{abstract}

Keywords. Magnetic fields, magnetohydrosynamics, Sun: corona, stars: coronae

\section{Introduction}

Owing to the low plasma $\beta$ of the solar corona, the energy which is needed to power flares and loop heating is believed to be primarily provided by the magnetic fields. Those are rooted in the photosphere, not only in large sunspots, but also in smallerscale flux concentrations such as those in plage regions (e.g. the so called network elements) as well as in the quiet Sun (also involving granular and intergranular flux tubes). First, it is now well extablished that solar flares are caused by magnetic reconnection. Second, even though several alternative-current (AC) wave-based models have been proposed for coronal heating, observational arguments tend to favor directcurrent (DC) mechanisms (Mandrini et al. 2000), the latter also being related to magnetic reconnection. 
Understanding the global geometry of the magnetic field involved in reconnection is important, not only to infer potentially different fundamental reconnection regimes, but also when one wants to identify their potential occurence in observed events, when analyzed with magnetic field extrapolations (see Démoulin et al. 1997 and Wiegelmann 2011 for linear and non-linear force-free extrapolation methods). Two-dimensional (2D) models for magnetic reconnection at magnetic X-points have been long since studied. They have been extended to fully three-dimensional (3D) magnetic fiels configurations involving null points and separator field lines. Those magnetic configurations involve separatrix field lines or surfaces, across which the connectivity of field lines changes in a discontinuous way (see the review by Galsgaard 2011). General magnetic reconnection theory, however, does not require such topological singularities in 3D (Hesse \& Schindler 1988). In this context, Priest \& Forbes (1992) have first shown that the behavior of 2.5D magnetic field lines, reconnecting at a $2 \mathrm{D} \mathrm{X}$-point with a finite guide field perpendicular to the plane of the X-shaped separatrices, was different from standard separatrix reconnection: they argued in favor of a continuous flippage of the field lines across the remnants of the separatrices, instead of a classical cut-and-paste reconnection. Quasi-separatrix layers (QSLs), which are narrow volumes across which the magnetic field connectivity changes drastically, but in a continuous way, have then been developped as a likely geometrical extension of 2.5D flipping layers, and of 3D separatrices found in classical source models (Priest \& Démoulin 1995; Démoulin et al. 1996a; Restante et al. 2009).

An impressive wealth of results has been obtained over the last two decades on the physics of QSL reconnection, on its application to solar observations, and on its relevance to several numerical and laboratory experiments. Instead of going through an exhaustive review of those, this author has chosen to briefly describe a personnal selection of only a few key results, which illustrate that many QSL-related studies actually show striking common features. To some extent, those highlight some unity which emerges from these disctinct approaches of coronal heating and flaring, around the concept of QSLs.

\section{CS formation and magnetic reconnection in QSLs}

The mathematically correct way to define a QSL is through the computation of the so-called squashing factor $Q$, which is constant along field lines, and which somehow measures the magnetic connectivity gradients around this field line (see Titov et al. (2002) for extensive details). A simpler way to identify the presence of QSLs, in a given magnetic field configuration, is to integrate field lines starting from small segments at the photospheric boundary: the spreading of their conjugate footpoints, along a curve that is much longer than the segment, indicates the presence of a QSL.

Démoulin et al. (1996a) were the first to give analytical arguments for QSLs being natural places for current sheet (CS) formation, driven by any line-tied photospheric motions, just like true separatrices are. Several groups have argued both in favor and against this idea (see e.g. Milano et al. 1999 and Galsgaard et al. 2003), until Aulanier et al. (2005) proved it right, with a series of numerical MHD of bipolar potential fields created by four flux concentrations, which initially possessed thin current-free QSLs. It has then been showed that QSLs are the 3D geometrical layers which support magnetic flipping, i.e. 3D finite- $B$ reconnection (Aulanier et al. 2006; see Figure 2). Slip-running reconnection was there defined as the regime for which the slippage velocity of field lines (which does not correspond to a mass motion) is super-Alfvénic (which happens for high enough $Q$ and reconnection rate). This regime cannot be misinterpreted as 


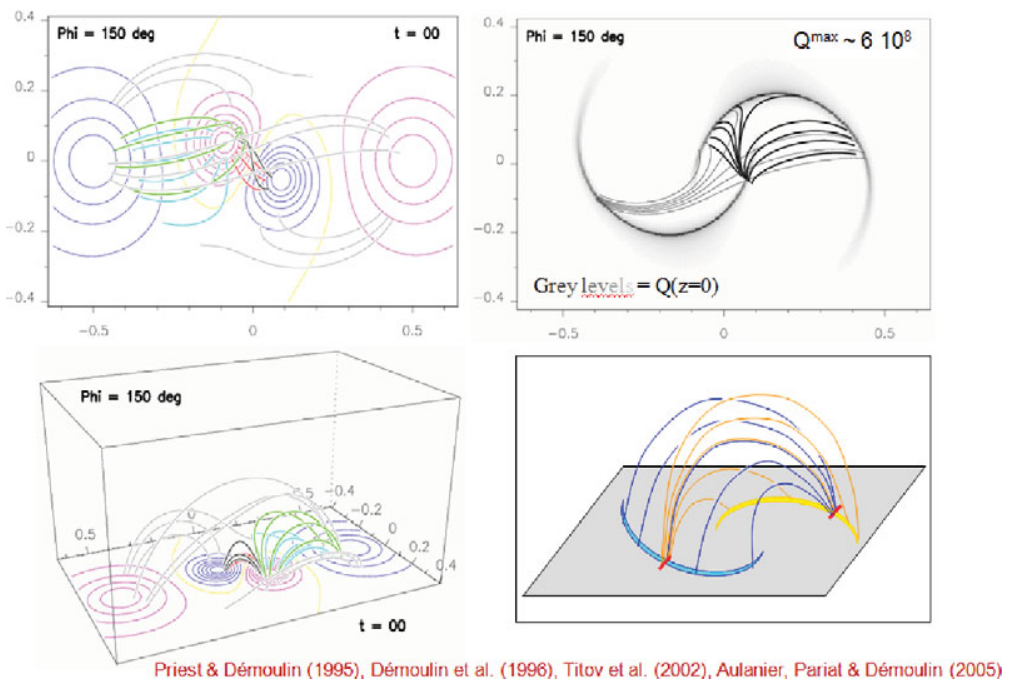

Figure 1. Example of QSLs formed in a 3D bipolar potential field, created by four flux concentrations. Note how field lines rooted in small segments, that cross high- $Q$ photospheric regions (i.e. QSL footpoints), spread out and map the other QSL footpoint.
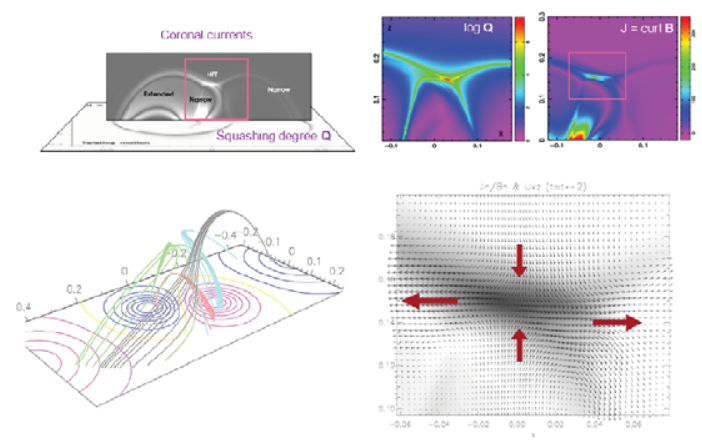

Aulanere, Panat, Démoulin \& DeVore (2006)

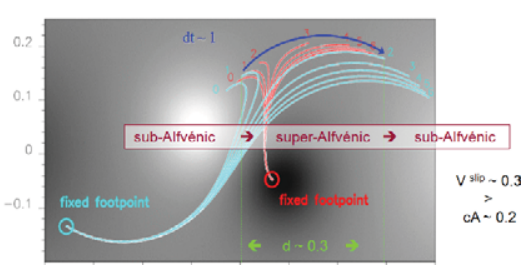

Figure 2. QSL related current sheet, that leads to finite- $B$ magnetic reconnection. When analyzed in $2 \mathrm{D}$, the standard X-type configuration and outflow jets are recovered, but the $3 \mathrm{D}$ picture shows a continuous slippage of field lines, which shows a slip-running regime which differs from mere diffusion.

magnetic diffusion, since it is local, and physically undistinguishable from mere null point or separator reconnection as far as MHD processes are concerned.

\section{Observational evidences of QSLs in the Sun's corona}

Before CS formation and reconnection was shown to occur in QSLs with MHD simulations, linear force-free field extrapolations of the coronal magnetic field, from line-of-sight 


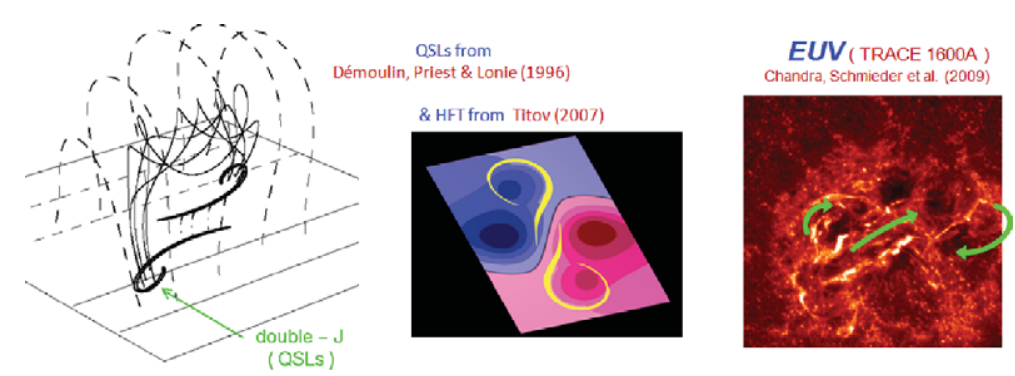

Figure 3. Double-J shaped QSL footprints in models consisting of a twisted flux tube embedded in a potential arcade, compared with a double-J shaped two-ribbon flare observed in the EUV.

magnetic field measurements in the photosphere, already brought strong hints about the role of QSLs in solar non-eruptive flares. More specifically, it has been shown that QSL footprints nicely match flare ribbons, which are elongated chromospheric brightenings observed in $\mathrm{H} \alpha$ and in the EUV, that result from the impact of particles accelerated in the corona during the flare (see e.g. Démoulin et al. 1997; Schmieder et al. 1997).

The role of QSLs in eruptive and so-called two-ribbon flares is more elusive, essentially due to the difficulty in calculating non-linear force-free extrapolations, that are required to recover the pre-eruptive highly current-carrying bipolar fields embedded in nearly potential arcades. Two arguments, however, suggest that QSLs are also involved in such events. Firstly, topological analyses of such configurations have been performed for purely theoretical models. Double-J shaped QSL footprints have been found to occur systematically around moderately (as well as strongly) twisted flux tubes (Démoulin et al. 1996b; Titov 2007; see Figure 3). Once knowing this, flare ribbons having such double-J shapes as recorded by various solar instruments can be identified in many observed eruptive events. One of the clearest cases, so far, has been reported by Chandra et al. (2009), and is shown in Figure 3.

Secondly, high-cadence HXR and EUV observations have revealed that strong brightenings are often seen to quickly propagate along eruptive flare ribbons (see Fletcher \& Hudson. 2002 and Bogachev et al. 2005). While this can be interpreted in terms of a 2D moving reconnection site in the corona, or by sequences of $2 \mathrm{D}$ tether-cutting reconnections involving arcades with different shears from one another, it is also compatible with the gradual formation of elongated channels of propapations for accelerated particles, associated with slipping and slip-running field lines, hence with QSL reconnection. Such tether-cutting slip-running reconnection has indeed been found in MHD simulations of solar eruptions (Aulanier et al. 2010; Fan 2010). 

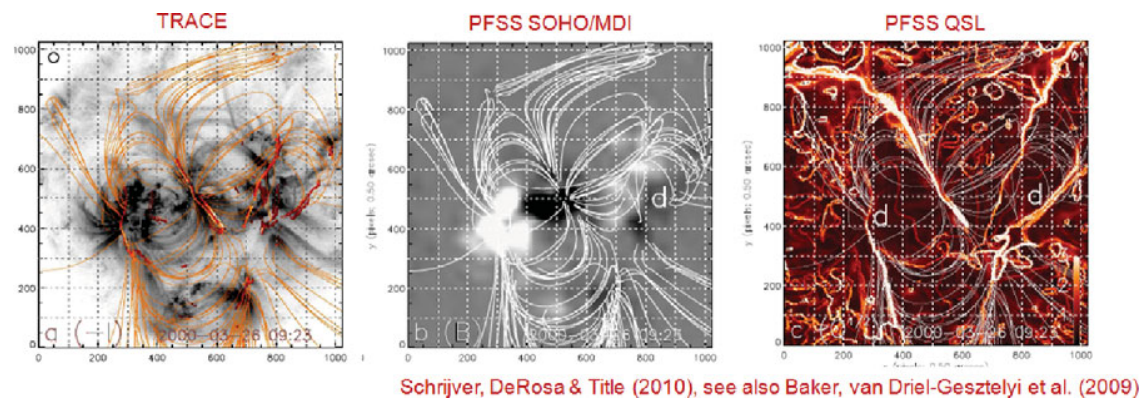

Figure 4. Warm EUV loops associated with QSL footpoints in a current-free magnetic field.

QSLs have also been associated with relatively quiet, non flaring, coronal loops in active regions, e.g. by combining magnetic field extrapolations and EUV or SXR observations.

Fletcher et al. (2001) associated Mg IX (resp. Si XII) emissions with QSL footprints (resp. loops), and concluded that transition region (resp. coronal) bright emissions could be associated with QSL heating. Schrijver et al. (2010) later identified that large-scale, current-free and warm coronal loops observed in Fe x, which interconnected several flux concentrations in active complexes, were typically rooted in QSL footprints (see Figure 4). They concluded that coronal heating should there be located at low heights (in accordance with current ideas of coronal heating in warm loops), since field line crossings (and therefore CS) produced by photospheric motions should remain confined at low altitudes, owing to the divergence with height of QSL flux tubes. Aulanier et al. (2007) reported on a rare observation of apparent bidirectional slipping of hot coronal loops observed in SXR, which arguably ressembles slipping and slip-running reconnection in QSL, and therefore concluded that QSLs may also play a role in the heating of hot active region loops. Baker et al. (2009) related the location of QSL footprints to the edge of large flow areas identified with Fe XII dopplershifts, and concluded that magnetic reconnection in QSLs could also be responsible for these hot coronal outflows, which are now typically observed on the edges of active regions.

\section{Are there QSLs in braided homogeneous and complex flux tubes?}

One of the main DC model for coronal heating is the Parker model, in which continuously and slowly braided field lines, in an initially homogeneous magnetic field, produce numerous CS whose dissipation produces heating. Over the years, this process was modeled with MHD simulations with larger and larger Reynolds numbers, first finding laminar/steady (van Ballegooijen 1986; Mikic 1989) and later turbulent/transient (Gómez et al. 1995; Hendrix \& van Hoven 1996; Rappazzo et al. 2008) CS.

The question of the geometrical properties of the magnetic field in these models was addressed by Milano et al. (1999). They found that two simple rotational footpoint mo- 

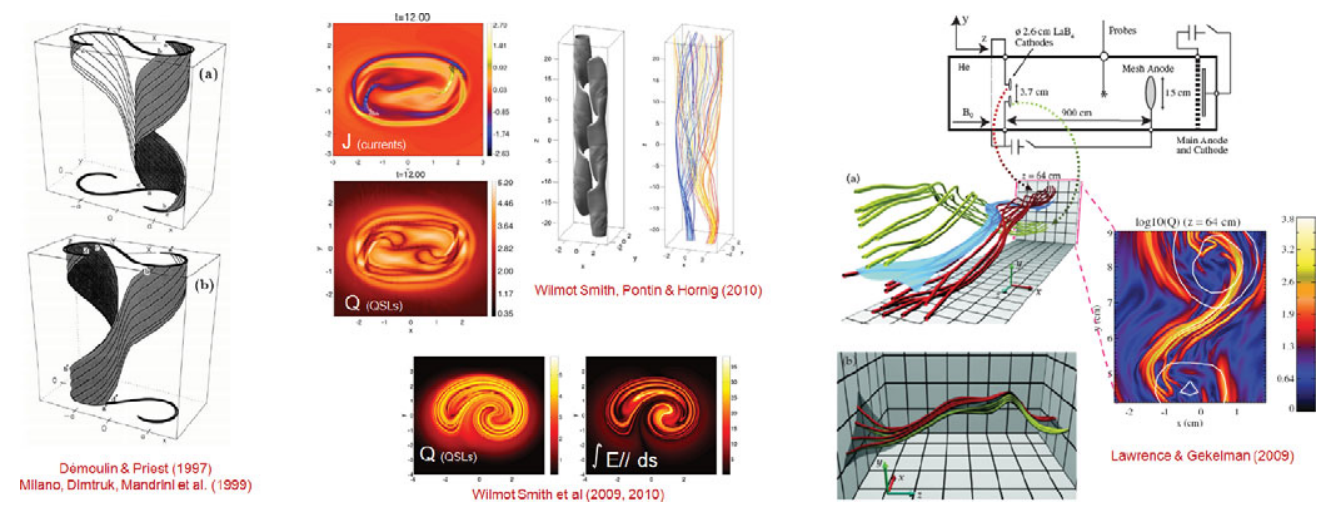

Figure 5. QSL located around and at the boundary between two twisted flux tubes (left), and QSL-related CS found with interacting twisted flux tubes, being produced numerically by boundary driving (middle) and experimentally by current injection (right).

tions, with a stagnation point in between, produce one single QSL-related S-shaped CS, at the boundary between the twisting flux tubes (in accordance with analytical predictions by Priest \& Démoulin 1997). This CS eventually becomes turbulent as reconnection there proceeds. This association was somehow recovered in different numerical expermiments (Wilmot-Smithet al. 2009, 2010), which found a good match between the locations of large $Q$ values and of strong currents, or integrated parallel electric fields, depending on the model. A QSL-related CS was also directly measured in a laboratory experiment of two interacting twisted flux tubes, there produced by a prescribed electric current instead of by footpoint motions (Lawrence \& Gekelman 2009).

These results, which are illustrated in Figure 5, tend to argue in favor of an important role of QSLs in turbulent braided field with very large Reynolds numbers, which can exist in the Sun's and in star's coronae (see e.g. Rappazzo et al. 2008), hence in coronal heating at yet-unobservable subscales inside individual coronal loops.

At moderately larger scale, it is known that magnetoconvection typically produces numerous and slowly evolving flux concentrations in the photosphere. These not only lead to a departure from the homogeneous magnetic field models described above, but also to a complex magnetic field pattern (sometimes called the magnetic carpet, observed both in the quiet Sun and inside active region faculae). Such complex magnetic fields ought to result in numerous QSLs, hence in numerous CS when driven by any smooth photospheric motion. In this picture, Figures 1 and 2 only show a mild idea of the complex QSL and CS patterns that can exist in the real Sun's atmosphere. Such complex QSL patterns, here shown in Figure 6, were first modeled by Priest \& Démoulin (1997), and later studied by Restante et al. (2009).

While the results of Schrijver et al. (2010) indicate that such complex QSL patterns can indeed be related to EUV coronal loops, quantifying their role in the real coronal heating process in the Sun, and in the one modeled in braiding simulations of action region magnetic fields obtained from force-free extrapolations (Gudiksen \& Nordlund 2005; see Figure 6), will still require more investigation. 

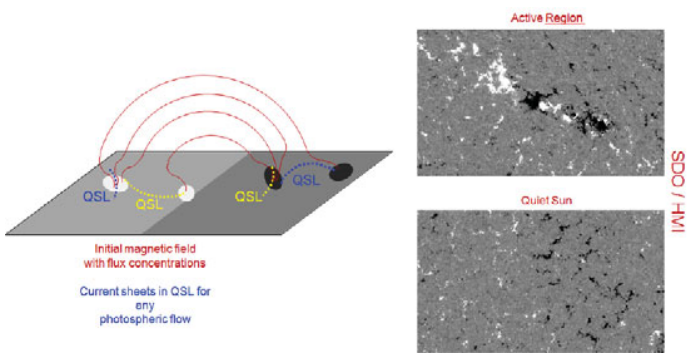
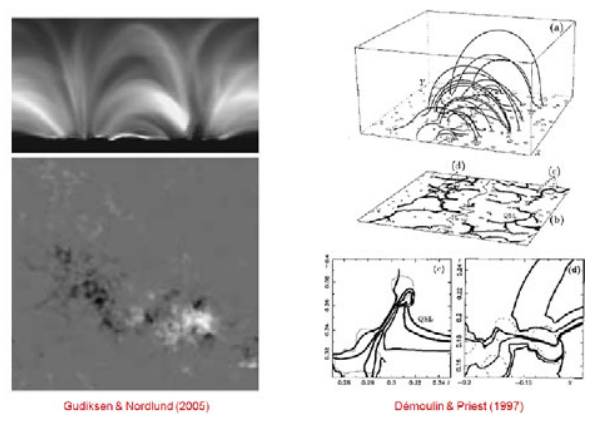

Figure 6. Observed complex flux concentrations (2nd column), resulting in QSLs, as hinted from geometrical arguments (1st column) indeed found in potential field models (4th column), and maybe occuring in MHD simulations of braided active regions (3rd column).

\section{Discussion}

QSLs are narrow layers of large spatial variations for the connectivity of magnetic field lines. QSLs naturally occur both in complex (but not necessarily multipolar) surface magnetic field distributions, and in the presence of large-scale volumetric fieldaligned electric currents, that produce localized (but not necessarily large) magnetic twist.

The current highest-resolution photospheric magnetic field measurements and coronal images (such as those of the Hinode, SDO and HiRISE) show that both situations indeed exist at various scales in the Sun's atmosphere: close to sunspots, between or inside active regions, and also in the quiet Sun. Several studies illustrated in this paper, when put all together, argue in favor of the ubiquituous existence of QSLs, and of their associated slipping and slip-running reconnection regimes, in the heating of solar coronal loops and in driving reconnection in solar flares. By extension, it is also arguable that the dissipation regions which occur in line-tied MHD turbulence (Gudiksen \& Nordlund 2005; Rappazzo et al. 2008) also correspond to QSLs. If so however, the causal relationship between QSL reconnections and the turbulent cascade will have to be analyzed.

Can these results be applied to star's coronae? The reliability of the Zeeman Doppler Imaging (ZDI) mapping of stellar surface magnetic fields is sill debated (see e.g. Carroll 2011). However, ZDI has recently provided impressive results, e.g. using the ESPaDOnS and NARVAL instruments (see e.g. Jardine et al. 2011). The obtained maps often show complex magnetic field distributions, which do fit the geometrical requirements for QSLs to exist. QSLs may therefore also be involved in the heating of some stellar's coronae. Testing this conjecture might be achieved through calculating QSLs and their localized enhanced EUV or X-ray emissions, using magnetic field extrapolations from ZDI maps, and comparing them with observed light curves as the stars rotate.

One may finally address the issue of the physical difference between QSLs and separatrices, as far as MHD processes only are concerned. First, CS naturally form in QSLs in the same way as they do in separatrices (Aulanier et al. 2005). Second, the slip-running reconnection regime in QSLs leads to super-Alfvénic field lines motions, which cannot be perceived by the magnetized plasma (Aulanier et al. 2006). Third, even though the 
existence of QSLs does not require the existence of 3D null points, QSLs also exist in the form of halos surrounding asymmetric null point separatrices, which there also sustain QSL reconnection regimes (Masson et al. 2009, submitted). These three issues imply that, physically, the magnetized plasma responds in very similar ways to CS formation and reconnection, and therefore heating and flaring, in QSLs as well as in null points. So, even though both features provide interesting mathematical tools for investigation, maybe should they start to be considered all together when studying MHD processes. Separatrices would then be considered as the limiting case of QSLs for which $Q \mapsto \infty$, as advocated by Démoulin (2006).

\section{References}

Aulanier, G., Pariat, E., \& Démoulin, P. 2005, Astron. Astrophys, 444, 961

Aulanier, G., Pariat, E., Démoulin, P., \& DeVore, C. R. 2006, Solar Phys., 238, 347

Aulanier, G., Golub, L., DeLuca, E. E., Cirtain, J. W., \& Kano, R., et al. 2007, Science, 318, 1588

Aulanier, G., Török, T., \& Démoulin, P., DeLuca E. E. 2010, Astrophys. J., 708, 314

Baker, D., van Driel-Gesztelyi, L., \& Mandrini, C. H., et al. 2009, Astrophys. J., 705, 926

Bogachev, S. A., Somov, B. V., \& Kosugi, T. Sakao, T. 2005, Astrophys. J., 630, 561

Carroll, T. A. 2011, Proceedinds of the IAU Symposium 273, this issue

Chandra, R., Schmieder, B., Aulanier, G., \& Malherbe, J.-M. 2009, Solar Phys., 258, 53

Démoulin, P., Hénoux, J.-C., Priest, E. R., Mandrini, C.H. 1996a Astron. Astrophys, 308, 643

Démoulin, P., Priest, E.R., Lonie, D. P. 1996b JGR, 101, A4, 7631

Démoulin, P., Bagala, L.G., Mandrini, C.H., Hénoux, J.C., Rovira, M.G. 1997 Astron. Astrophys, 325,305

Démoulin, P. 2006 Adv. Sp. Res., 37, 7, 1269

Fan, Y. 2010, Astrophys. J., 719, 728

Fletcher, L., López Fuentes, M. C., \& Mandrini, C. H., et al. 2001, Solar Phys., 203, 255

Fletcher, L. \& Hudson, H. S. 2002, Solar Phys., 210, 307

Galsgaard, K., Titov, V. S., \& Neukirch, T. 2003, Astrophys. J., 595, 506

Galsgaard, K. 2011, Proceedinds of the IAU Symposium 273, this issue

Gómez, D. O., DeLuca, E. E., \& McClymont, A. N. 1995, Astrophys. J., 448, 954

Gudiksen, B. V. \& Nordlund, A. 2005, Astrophys. J., 618, 1031

Hendrix, D. L. \& van Hoven, G. 1996, Astrophys. J., 467, 887

Hesse, M., Schindler, K. 1988 JGR, 93, 5559

Jardine, M., Donati, J.-F. et al. 2011 Proceedinds of the IAU Symposium 273, this issue

Lawrence, E. E. \& Gekelman, W. 2009, PRL, 103, 10, 105002

Mandrini, C.H., Démoulin, P., Klimchuk, J.A. 2000 Astrophys. J., 530, 999

Masson, S., Pariat, E., Aulanier, G., \& Schrijver, C. J. 2009, Astrophys. J., 700, 559

Masson, S., Aulanier, G., Pariat, E., \& Klein, K. L. 2010, Astrophys. J. (Letters), submitted

Mikic, Z., Schnack, D. D., \& van Hoven, G., 1989, Astrophys. J., 338, 1148

Milano, L. J., Dmitruk, P., Mandrini C. H., Gómez, D. O., \& Dmoulin, P. 1999, Astrophys. J., 521,889

Priest, E. R. \& Forbes, T. G. 1992, JGR, 97, A12, 1521

Priest, E. R. \& Démoulin, P. 1995, JGR, 100, A12, 23443

Priest, E. R. \& Démoulin, P. 1997, Solar Phys., 175, 123

Rappazzo, A.F., Velli, M., Einaudi, G., Dahlburg, R.B. 2008 Astrophys. J., 677, 1348

Restante, A. L., Aulanier, G., \& Parnell, C. E. 2009, Astron. Astrophys, 508, 433

Schmieder, B., Aulanier, G., Démoulin, P., \& van Driel-Gesztelyi, L., et al. 1997, Astron. Astrophys, 325, 1213

Schrijver, C. J., DeRosa, M. L., \& Title, A. M. 2010, Astrophys. J., 719, 1083

Titov, V. S. 2007, Astrophys. J., 660, 863 
Titov, V. S., Hornig, G., \& Démoulin, P. 2002, JGR, 107, A8, SSH 3-1 van Ballegooijen, A. A. 1986, Astrophys. J., 311, 1001

Wiegelmann, T. 2011, Proceedinds of the IAU Symposium 273, this issue

Wilmot-Smith, A. L., Hornig, G., \& Pontin, D. I. 2009, Astrophys. J., 696, 1339

Wilmot-Smith, A. L., Pontin, D. I., \& Hornig, G. 2010, Astron. Astrophys, 516, 5 